05

\title{
Моделирование механических свойств сильно неоднородных систем под внешним воздействием
}

\author{
() П.А. Александров, ${ }^{1}$ А.Б. Свечников, ${ }^{1}$ B.B. Горев, ${ }^{1}$ E.V. Ryan, ${ }^{2}$ W.H. Ryan, ${ }^{2}$ H.K. Stange-Love ${ }^{2}$ \\ ${ }^{1}$ Национальный исследовательский центр „Курчатовский институт“, \\ 123182 Москва, Россия \\ ${ }^{2}$ New Mexico Institute of Mining \& Technology, \\ NM 87801 Socorro, USA \\ e-mail: Alexandrov_PA@nrcki.ru
}

Поступило в Редакцию 17 января 2019 г.

В окончательной редакции 17 января 2019 r.

Принято к публикации 20 февраля 2019 г.

\begin{abstract}
Проведены лабораторные испытания, дополненные новой числовой моделью, для изучения механических свойств астероидов с сильно неоднородной структурой. В числовой модели использована функциональная форма метода молекулярной динамики, расширенной до макроскопических пространственных масштабов. В эксперименте мишени, состоящие из стеклянных шариков, скрепленных обычным льдом, взаимодействовали с быстрыми импакторами. Высокоскоростными камерами зафиксированы процесс разрушения матрицы льда и сохранение стеклянных шариков без повреждений. Параллельно выполнена параметризация математической модели, полученные расчетные данные сравнены с экспериментом. Удалось определить область энергий импакторов, когда удар вызовет отклонение мишени при минимальном разрушении.
\end{abstract}

DOI: $10.21883 /$ JTF.2019.07.47800.9-19

\section{Введение}

Подробные исследования астероидов и комет вместе с их столкновительной динамикой дают фундаментальные данные, необходимые для понимания физических процессов, которые формировали нашу солнечную систему и определяли ее последующую эволюцию. Знания в области физики столкновений определяют понимание планетарного роста в процессе формирования солнечной системы, ее последующей эволюции с включением динамических и негравитационных механизмов доставки метеоритов. Эти процессы непосредственно влияют на наблюдаемое размерно-частотное распределение пояса астероидов, которое позволяет нам измерять законы масштабирования сил для этих планетарных строительных блоков, минералогическую структуру пояса астероидов и возникновение бинарных астероидных систем, которые определяются физическими и морфологическими свойствами предков и воздействием астероидов.

Ученые-планетологи добились огромного прогресса благодаря лабораторным экспериментам по соударениям тел, постоянному совершенствованию численных расчетов динамической и физической эволюции солнечной системы, наземным и космическим наблюдениям. Теоретическая и лабораторная работа показала, как астероиды взаимодействуют через столкновения и как на них влияют солнечные радиационные силы, гравитационные моменты и другие процессы. Телескопические исследования дали параметры орбит для почти миллиона астероидов, спектроскопические исследования позволили получить данные об их составе, области образования и размерах, и, наконец, фотометрические исследования позволили определить формы и скорости вращения.

Практический интерес к этой теме обусловлен главным образом наличием постоянной угрозы столкновения астероидов с Землей [1]. Разработка методов воздействия на астероиды сильно зависит от знания внутренней структуры и состава астероида. Ценные данные получены при изучении метеоритов, поскольку эти объекты способны проникать через атмосферу. По оценкам, лишь 7\% каменных объектов достигают поверхности Земли без полного разрушения, и, как следствие, пока не существует образца, имеющего достоверный состав. Кроме того, не только состав может влиять на результаты столкновений, надо учитывать высокую пористость структуры астероидов. Считается, что многие астероиды с низкой плотностью являются скоплениями щебня, это относится и к кометам. Наблюдения кометы Хартли-2 показали, что на ее поверхности существует область, напоминающая структуру гантели, соединенную гладкой соединительной шейкой без кратеров. Как было предложено авторами [2], эта морфология представляет собой медленный распад ядра кометы на две части с соединительной горловиной в результате постоянного действия центробежных сил. Особенности текстуры в области шейки (где отсутствуют какие-либо ударные кратеры или выступающие камни) объясняется постоянным восстановлением поверхности, возможным только в случае слабосвязанных мелких компонентов. Эти композиционные подсказки помогают в разработке исследовательской программы для изучения фрагментации астероидов и, в конечном итоге, подходов, которые 
могут быть полезными при разработке методов отклонения объектов, сближающихся с Землей.

Целью настоящей работы является использование экспериментов по столкновениям тел и нового подхода к численному моделированию динамики астероидов с неоднородными, слабосвязанными структурами. В числовой модели используется функциональная форма метода молекулярной динамики (МД) в макроскопических пространственных масштабах. Этот подход был ранее продемонстрирован успешным моделированием явлений геологического разлома $[3,4]$. Следующий раздел посвящен методике получения мелкомасштабных, сильнонеоднородных и слабосвязанных конгломератов, которые затем использовались в качестве мишеней. В состав мишени входили стеклянные шарики разных размеров и некоторое количество льда. Мишень получала удар после выстрела импакторами, число и энергия которых варьировалась. Разрушение мишени на отдельные фрагменты происходило по матрице льда и стеклянные шарики не повреждались. Затем рассматривается математическая модель динамики столкновений, основанная на приближении парных потенциалов взаимодействия между шариками и импакторами. Как следует из представленных в конце численных результатов, модель хорошо описывает наблюдаемую динамику разрушения мишени.

\section{1. Мишени для экспериментов по рассеянию}

Ранее исследователи [5] использовали способ получения пористых структур определенной формы путем спекания стеклянных шариков при высоких температуpax. В качестве более простой альтернативы спеканию мы используем обычный лед в качестве связующего материала. Стеклянные шарики диаметром от 10 до $20 \mathrm{~mm}$ помещают в сетку сферической формы и замораживают до температуры от -30 до $-45^{\circ} \mathrm{C}$. Затем сетку с шариками погружают на несколько секунд в емкость с водой при температуре $3^{\circ} \mathrm{C}$. В результате образуется сплошная сферическая мишень из стеклянных шариков, связанных между собой тонкими ледяными мостиками. После удаления сетки мишень может долго храниться в контейнере, заполненном сухим льдом. Во время эксперимента ледяная матрица стабильна в течение $20 \mathrm{~min}$ при комнатной температуре. К преимуществам заморозки можно отнести простоту изготовления, повторное использование стеклянных компонентов и, следовательно, низкую стоимость производства мишеней.

Поскольку образование периодических структур из шариков рассматривалось нами как негативный фактор, в эксперименте были задействованы шарики трех разных размеров, и соответствующее количество дефектов в мишени приближалось к числу шариков. Можно сделать предположение о характере разрушения мишени при внешнем механическом воздействии, а именно через

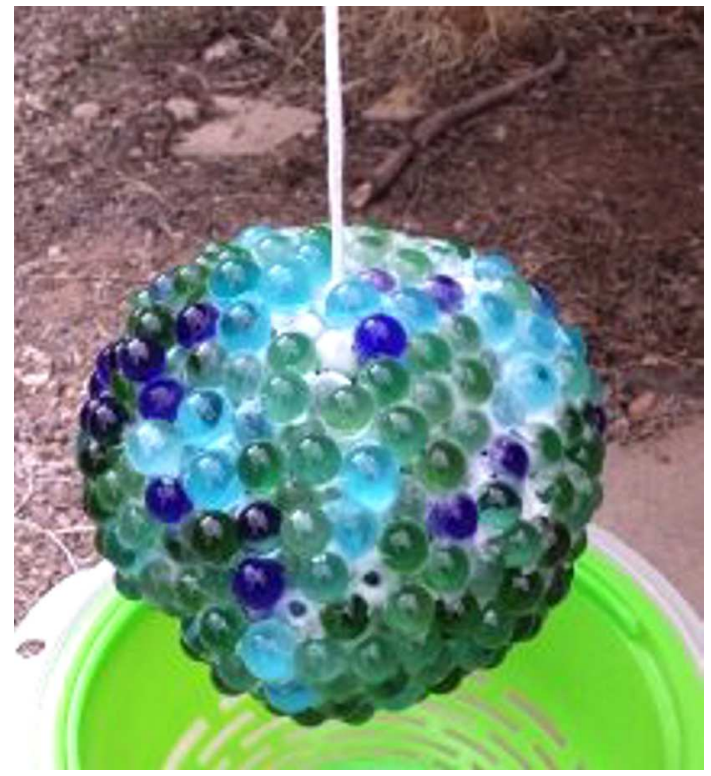

Рис. 1. Мишень из стеклянных шариков, скрепленных льдом.

накопление напряжений и последующий разрыв ледяных мостиков в местах скопления структурных дефектов, где расстояния между шариками превышают среднюю величину.

Для создания мишеней, каждая по $3.5 \mathrm{~kg}$ в массе и примерно $16 \mathrm{~cm}$ в диаметре, всего смешивались примерно 2700 стеклянных шариков трех размеров (с цветовой кодировкой). И для конкретных мишеней использовалось примерно 900 штук (рис. 1). Поверхности шариков имели незначительные дефекты, которые позволяли увеличить сцепление со льдом по сравнению с совершенно гладкими шариками.

Полученные мишени имели среднюю объемную плотность $1.5 \mathrm{~g} / \mathrm{cm}^{3}$ со средней пористостью $36 \%$, что соответствует характеристикам известных астероидов, полученным путем измерений и оценок.

\section{2. Эксперименты с предварительным воздействием}

Чтобы проверить является ли представленная модель мишени аналогом слабосвязанных астероидов, было проведено несколько предварительных экспериментов. Прочность на растяжение измерялась для системы из двух стеклянных шариков с мостиком между ними. Было обнаружено, что это значение очень близко к известной когезионной прочности льда при данной температуре, следовательно, энергия когезии ниже энергии адгезии. Следует отметить, что энергия сцепления сильно зависит от чистоты поверхности, поэтому масло и другие загрязняющие вещества были удалены с поверхностей стеклянных шариков прежде, чем создавались ледяные мостики и выполнялись измерения. Эксперименты про- 
водились для тетраэдра из четырех стеклянных шариков. Прочность на растяжение льда при температуре $-10^{\circ} \mathrm{C}$ была определена как $10 \mathrm{~kg} / \mathrm{cm}^{2}$, что соответствует стандартным значениям для льда. Данный результат был использован в последующих экспериментах с мишенями, чья температура поддерживалась ниже $-20^{\circ} \mathrm{C}$. Понижению температуры соответствует плоский участок со значением силы на разрыв $16 \mathrm{~kg} / \mathrm{cm}^{2}$, тогда как у стекла эта величина значительно больше $-1400 \mathrm{~kg} / \mathrm{cm}^{2}$ [6].

В следующем эксперименте участвовала мишень диаметром $16 \mathrm{~cm}$, содержащим стеклянные шарики следующих диаметров (заданные в сm): 1.36 (55\%), 1.40 (22\%), 1.62 (23\%); процент шариков, включенных в цель, указан в скобках. Шарики удерживались вместе с помощью тонкой сетки, лед отсутствовал. Мишень подвешивалась на веревке, прикрепленной к сетке, и примерно в середину мишени сбоку ударялся импактор из полипропилена диаметром $0.63 \mathrm{~cm}$, весом $0.119 \mathrm{~g}$, со скоростью $607 \mathrm{~m} / \mathrm{s}$. Целью этого опыта было определение количества расколовшихся стеклянных шариков. В результате оказалось, что количество расколовшихся шариков составляет менее $1 \%$ от общего количества в образце. По всей видимости, раскололись шарики только при прямом контакте с импактором. Таким образом, кинетическая энергия импактора на разрушение шариков практически не расходовалась, и в дальнейшем мы будем принимать, что вся энергия пошла на смещение шариков мишени и приобретение ими скорости.

\section{3. Эксперименты и результаты}

Три эксперимента было проведено с мишенями из стеклянных шариков, удерживаемых вместе ледяной матрицей. Процентный состав шариков разного диаметра в мишенях оставался таким же, как и для предварительного эксперимента без льда. Смесь стеклянных шариков замораживалась в сетке при температуре $-32^{\circ} \mathrm{C}$, термопара в центре образца показала $-23^{\circ} \mathrm{C}$. Затем сетку погружали на короткое время (несколько секунд) в ванну с ледяной водой при температуре около $3^{\circ} \mathrm{C}$. После этого сетка снималась, и образец помещался в термостат с „сухим льдом“, где мог храниться сутками. Внутрь образца помещалась тонкая веревка, которая вмораживалась в образец и служила для его подвески во время эксперимента.

Эксперименты проводились на полигоне Института горного дела и технологий в Нью-Мексико (NMT) Научно-испытательного центра энергетических материалов (EMRTC) с использованием пушки. Установка показана на рис. 2.

В висящий образец производился выстрел, в результате которого описанный выше импактор попадал примерно в центр образца, имеющего форму шара, со скоростями $613,519,511 \mathrm{~m} / \mathrm{s}$ в каждом опыте соответственно. Процесс разрушения фиксировался высокоскоростной камерой (10000 fps) Phantom. Было отмече-

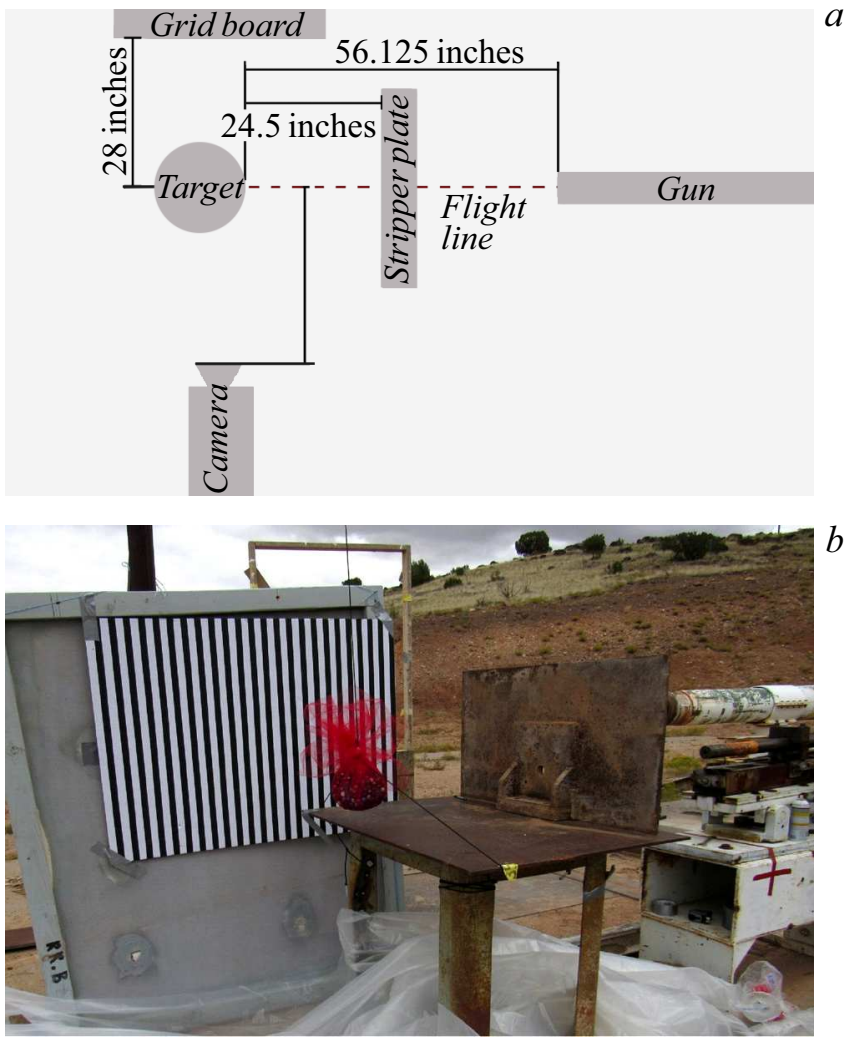

Рис. 2. Накладная диаграмма экспериментальной установки $(a)$ и фактическая установка перед стрельбой $(b)$, справа виден ствол пушки.

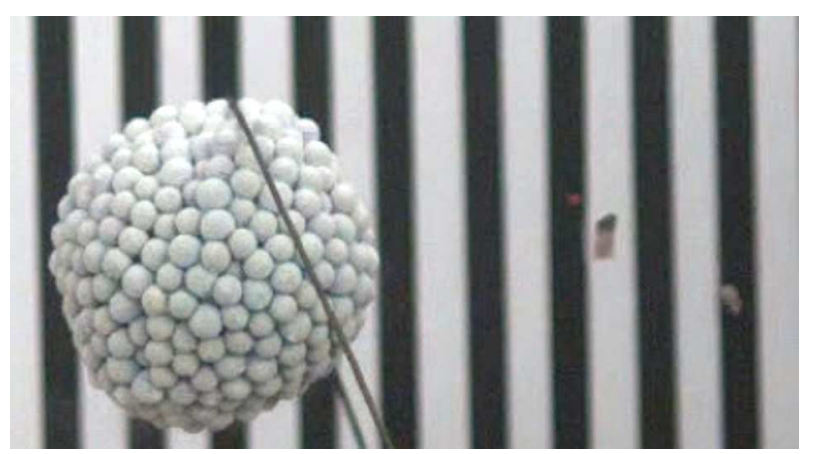

Рис. 3. Мишень и импакторы (справа) в эксперименте № 2.

но, что количество расколовшихся шариков в каждом эксперименте менее 1\% (всего несколько штук). Было получено распределение фрагментов по скоростям разлета и по массам, которые для трех опытов оказались очень близкими. Далее сравнивался с расчетами опыт № 2. При тщательном рассмотрении процесса удара импактора в объект оказалось (рис. 3), что вместе с импактором в объект ударялись два фрагмента пыжа, которые по случайным обстоятельствам пролетели сквозь диафрагму, специально поставленную между объектом и стреляющим устройством. Скорости двух примерно 


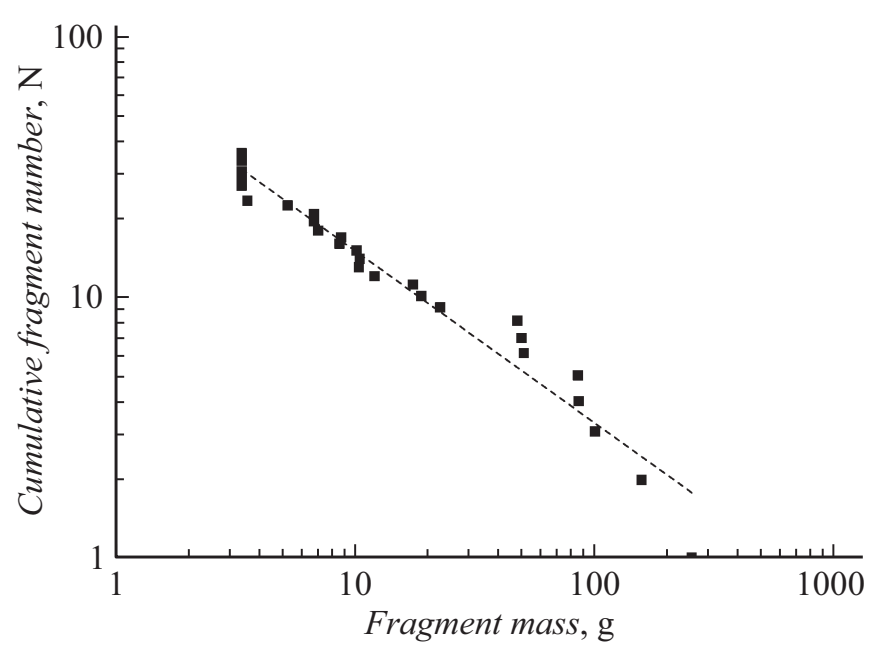

Рис. 4. Результирующие массовые распределения фрагментов (экспериментальные данные показаны точками). Кумулятивное число - это количество фрагментов данной массы или больше.
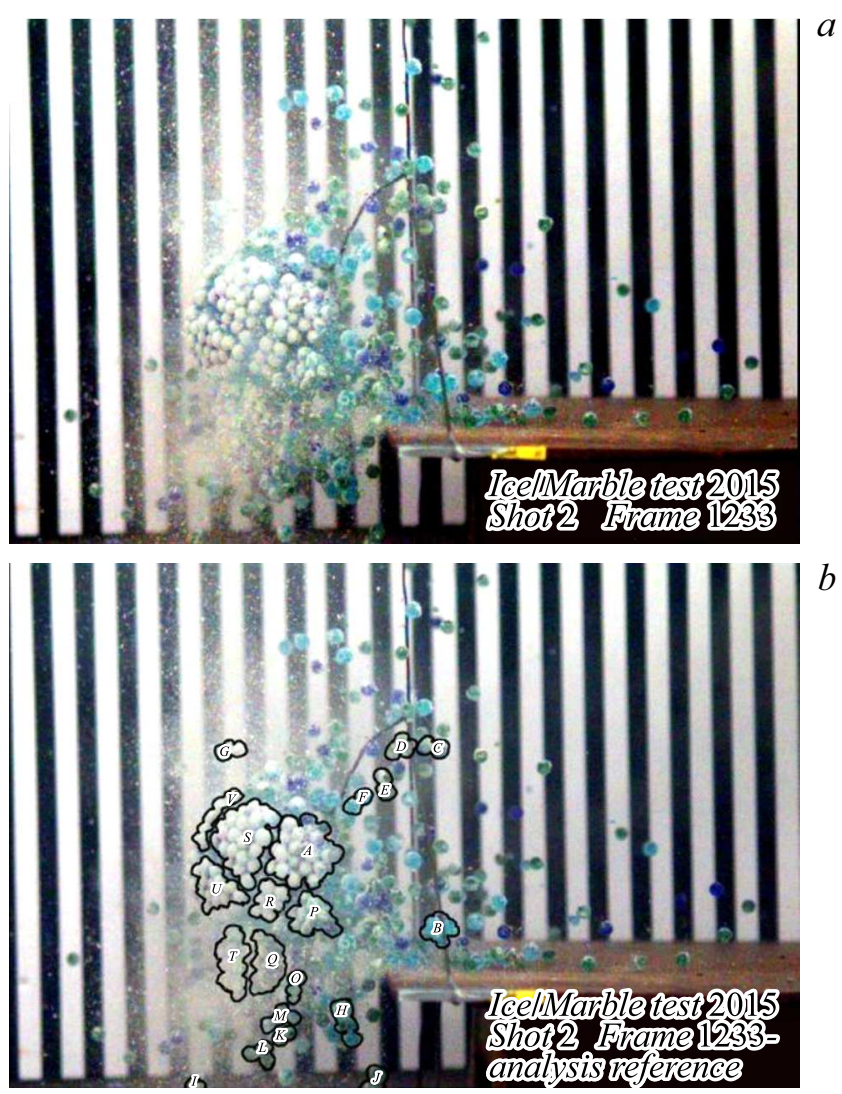

Рис. 5. Анализ фрагментов мишени после удара с помощью видеозаписи эксперимента № 2.

равных по размеру фрагментов пыжа оказались равными 511 и $461 \mathrm{~m} / \mathrm{s}$, их энергия при массе каждого $1.45 \mathrm{~g}$ (масса всего пыжа $2.9 \mathrm{~g}$ ) оказалась 190.3 и $157.5 \mathrm{~J}$, т.е. значительно больше, чем энергия импактора, равная $16.5 \mathrm{~J}$.
Кроме того, были проблемы с созданием мишени для эксперимента № 3, когда подвесной шнур изначально не был заморожен достаточно глубоко, чтобы удерживать вес. Он был залатан дополнительной ледяной водой и хорошо удерживался до удара. Однако дополнительный лед создал облако при ударе, что затруднило визуальный анализ фрагментов. В итоге эксперимент № 2 был выбран для детального анализа и для последующего сравнения с численной моделью. Распределение масс фрагментов мишени (рис. 4) было получено путем анализа изображений камеры (рис. 5).

Полученная зависимость согласуется с результатами по фрагментации горных пород, льдов и металлов [7], когда преобладали мелкие фрагменты и интерполяционная прямая имеет отрицательный $(-0.7)$ наклон. Проекции поступательных скоростей фрагментов на плоскость съемки в целом невелики (порядка $1 \mathrm{~m} / \mathrm{s}$ ), максимальное значение составляло $13 \mathrm{~m} / \mathrm{s}$. Медленные скорости фрагментов из эксперимента также согласуются с предыдущими результатами [7], когда не более 2\% кинетической энергии импактора переходит в поступательное движение фрагментов мишени, образованных в результате удара.

Ниже представлена численная модель исследуемого процесса, а полученные с ее помощью результаты сравниваются с результатами эксперимента № 2 .

\section{4. Моделирование процесса создания и разрушения мишени}

Теоретическая часть работы состояла в построении и тестировании численной модели столкновения импактора с мишенью для наилучшего воспроизведения представленных выше экспериментальных результатов. Из литературы известно [8,9], что моделирование таких систем может быть проведено методом гранулярной динамики. Предполагается, что многие параметры материала и неоднородности внутренней структуры мишени должны быть описаны и измерены, однако на практике они не всегда известны. Кроме того, для реализации этого метода требуются значительные вычислительные мощности. Группа авторов [10], которая также работает над изучением фрагментации слабосвязанных неоднородных астероидных структур, предложила объединить гранулярную динамику и парные потенциалы. Это экономит вычислительные ресурсы, но сохраняет другие недостатки. Например, не следует забывать о важности сил притяжения в задачах рассеяния и разрушения, ведь они обеспечивают стабильность и сопротивляемость многочастичной системы внешним воздействиям. Гранулярная динамика практически игнорирует эту специфику. Кроме того, нормальная компонента силы отталкивания может иметь негладкий вид, а это порождает неконтролируемые ошибки численного интегрирования уравнений движения. Еще одна неприятность - это коэффициент 
скользящего трения для расчета тангенциальной компоненты силы, он не может быть получен экспериментально. Все это значительно снижает качество физического представления.

Интересны результаты гидродинамических расчетов контактных взрывов для полномасштабных моделей астероидов [11]. Показано, что $6 \mathrm{Mt}$ взрыв приблизительно подобен импульсному нагреву миниатюрной мишени лазером с энергией $200 \mathrm{~J}$. Сделан вывод об избыточных затратах энергии для полного разрушения мишени. Математическая модель описывает мишень, как непрерывную среду, при этом численные методы используют разбиение среды на ячейки и временную дискретизацию уравнений.

В настоящей работе мы адаптировали метод молекулярной динамики (МД) для описания макроскопической системы, и не столкнулись с представленными выше трудностями. Как и в классическом методе МД, задаются парный потенциал взаимодействия шариков, начальное положение и скорость каждого шарика. Моменты инерции всех шариков игнорируются. Затем, решая уравнения Ньютона с известным потенциалом и массой, определяем новые координаты и скорости всех шариков через малое время $\Delta t$. Многократные проведения этой процедуры позволяют проследить эволюцию системы, и основной проблемой является выбор МД-потенциала.

\section{1. Выбор потенциала и его параметров}

Потенциал взаимодействия между отдельными частицами задает так называемое силовое поле, которое полностью определяет динамику системы с заданными начальными положениями и скоростями. Обычно к потенциалу предъявляют три основных требования: точность, универсальность и эффективность.

Точность потенциала обеспечивается путем оптимального выбора его параметров для наилучшего воспроизведения экспериментальных данных. Как показывает опыт, в природе не существует „хороших“ или „плохих“ потенциалов. В то же время необходимым условием выбора конкретного вида потенциала служит степень его соответствия решаемой задаче, которая устанавливается с помощью тестовых расчетов.

Далеко не все потенциалы являются универсальными, потенциал Леннарда-Джонса (Л-Д), использованный нами, является редким исключением из правила. Подтверждением этому служат многочисленные молекулярно-динамические расчеты газообразных систем, жидкостей, твердых тел, макромолекул и кластеров. При этом исследовались самые разные процессы: фазовые переходы, образование дефектов, рассеяние частиц и многие другие.

Потенциалы, зависящие только от расстояния между двумя взаимодействующими частицами, называются парными. Использование парных потенциалов в расчетах на современных вычислительных комплексах дает возможность исследовать очень крупные системы из $10^{7}-10^{10}$ частиц или прослеживать эволюцию меньших систем на длительных интервалах времени. Выражение для парного потенциала Л-Д представляет собой разность двух степенных функций расстояния и очень просто в вычислении

$$
U\left(r_{i j}\right)=4 \varepsilon\left[\left(\sigma / r_{i j}\right)^{12}-\left(\sigma / r_{i j}\right)^{6}\right] .
$$

Первое слагаемое $\sim 1 / r^{12}$ описывает отталкивание между частицами при их сильном сближении $\left(\sigma>r_{i j}\right)$. Степень 12 выбрана из соображений экономии вычислений. В других потенциалах аналогичное слагаемое часто выражают экспоненциальной функцией (потенциал Борна-Майера), однако это не имеет никакого принципиального преимущества.

Второе слагаемое $\sim 1 / r^{6}$ играет роль при удалении частиц друг от друга и обеспечивает их „связывание“ $\left(\sigma \leq r_{i j}\right)$. Традиционно эту часть трактуют как небольшой довесок, превращая тем самым потенциал Л-Д в „слабый“. Однако с математической точки зрения вид зависимости никак не связан с глубиной дна потенциала и полностью определяется „энергетическим“ параметром $\varepsilon$.

Выражения для производной потенциала Л-Д $\left(U^{\prime}\left(r_{i j}\right)\right)$ и силы, действующей на частицу $F_{i}$, имеют следующий вид:

$$
\begin{aligned}
& d U\left(r_{i j}\right) / d r_{i j}=4 \varepsilon\left(-12 \sigma^{12} / r_{i j}^{13}+6 \sigma^{6} / r_{i j}^{7}\right), \\
& F_{i}=-\sum_{j \neq i} 24 \frac{\varepsilon \sigma^{6}\left(r_{i}-r_{j}\right)}{r_{i j}^{8}}\left[1-2\left(\frac{\sigma}{r_{i j}}\right)^{6}\right] .
\end{aligned}
$$

Параметр $\sigma$ характеризует равновесное расстояние между связанными частицами

$$
U(\sigma)=0, \quad U\left(2^{1 / 6} \sigma\right)=U_{\min } .
$$

В нашем случае равновесным является положение, когда шарики касаются друг друга, и параметр $\sigma_{i j}$ вычисляется через диаметры шариков $\left(d_{i}, d_{j}\right)$

$$
\sigma_{i j}=2^{-7 / 6}\left(d_{i}+d_{j}\right)
$$

В точке $R_{\max }$, когда постоянная Хука становится равной нулю и сила Л-Д достигает максимума, имеем уравнение

$$
d^{2} U / d r^{2} \mid R_{\max }=0
$$

с решением

$$
R_{\max }=(13 / 7)^{1 / 6} R_{\min }=1.109\left(d_{i}+d_{j}\right) / 2 .
$$

Силы связи можно определить, если приравнять предел прочности Л-Д, решение (2) в $R_{\max }$ и экспериментальные значения для ледяных мостиков между стеклянными шариками (см. разд. 2). Разделив экспериментальные значения сопротивления разрыву на поперечное сечение льда, которое можно оценить как $\left(d_{i}^{2}+d_{j}^{2}\right) / 2$, без труда определяем энергетические параметры $\varepsilon_{i j}$ 
потенциала Л-Д. Например, для пары шариков диаметром $d_{1}=13.6$ и $d_{2}=16.2 \mathrm{~mm}$, соединенных квадратным монолитным ледяным мостиком размером $14.9 \mathrm{~mm}$ (при значении прочности на растяжение $11.3 \mathrm{~kg} / \mathrm{cm}^{2}$ ), соответствующие параметры Л-Д будут следующие: $\sigma_{12}=13.3 \mathrm{~mm}$ и $\varepsilon_{12}=1.3677 \mathrm{~J}$.

Однако в нашем случае лед не заполняет все пространство между бусинами. Взвешиванием было установлено, что в мишени, использованной в эксперименте № 2, лед заполнял лишь $18 \%$ от общего объема пустот. Следовательно, параметр энергии следует умножать на коэффициент заполнения льда (в данном случае 0.18 ).

\section{2. Подготовка мишени для численного моделирования}

На первом этапе компьютерного эксперимента требовалось сформировать геометрические модели мишеней, которые обладали достаточно высокой плотностью $(\sim 0.6)$ и были образованы заданным числом шариков трех типов. В литературе представлены алгоритмы решения подобных задач с использованием геометрических и статистических методов. Мы в настоящей работе провели апробацию двух вычислительных схем построения мишени на основе физических методов, а именно молекулярной динамики и Монте-Карло.

Последовательность выполнения Монте-Карло расчета была следующей. Область сферической формы заполнялась требуемым количеством шариков случайным образом. Для таких начальных структур из непересекающихся шариков характерна низкая плотность, порядка 0.3. Затем определялась потенциальная энергия взаимодействия $\left(U_{1}\right)$, равная сумме парных вкладов, рассчитанных с помощью потенциала Л-Д с параметрами для льда. После этого положения шариков изменялись случайным образом, и вычислялась потенциальная энергия $\left(U_{2}\right)$. Новую геометрию сохраняли для дальнейшего анализа при условии ее большей стабильности $\left(U_{2}<U_{1}\right)$. Процедуру поиска повторяли до достижения сходимости, $\left|U_{k^{-}} U_{k-1}\right|<\varepsilon$, где $\varepsilon=10^{-6} \mathrm{~J}$. Ускорить сходимость позволяло динамическое понижение амплитуд случайных смещений.

В методе МД выбор начального расположения шариков аналогичен описанному выше, и каждому шарику присваивалась скорость $|v|=0 \mathrm{~m} / \mathrm{s}$. Расчет состоял из циклического выполнения МД-шагов с фиксированным значением временного шага $\left(\Delta t=10^{-7} \mathrm{~s}\right)$. На основании потенциала Л-Д вычислялись силы взаимодействия между шариками. Затем путем интегрирования уравнений движения определялись новые положения и скорости для каждого шарика. С определенной периодичностью скорости всех частиц понижались на величину $10^{-5} \cdot|v|$. Окончание расчета происходило при полном „охлаждении“ системы, т.е. при $v \sim 0 \mathrm{~m} / \mathrm{s}$.

Для приведенных алгоритмов характерны приблизительно равные вычислительные затраты при расчете мишеней с числом шариков 900 штук.

\section{3. Расчет моделей}

Серия молекулярно-динамических расчетов проводилась для случайных начальных положений импакторов, на 20 различных мишенях. В общей сложности выполнено около 900 модельных расчетов с временным шагом $10^{-7} \mathrm{~s}$ и длительностью $10^{5}$ циклов. Благодаря представительной выборке удалось детально изучить динамику моделируемой системы и установить факт того, что выбранный потенциал Л-Д и соответствующий набор параметров хорошо воспроизводит экспериментальные данные.

На рис. 6 приведена конечная геометрия системы из модельного расчета $(a)$ и рядом для сравнения кадр из видео эксперимента $(b)$. Отметим высокое сходство изображений, из которого следует, что правая сторона мишени полностью разрушена в результате попадания импакторов. Образовавшиеся фрагменты имеют небольшие размеры, в основном это одиночные шарики. Левая сторона уцелела, но получила множественные дефекты и начинает разрушаться. Видно, что фрагменты экспериментальной мишени испытывали действие земного тяготения, а в численной модели им пренебрегли.

На рис. 7 представлены экспериментальные и расчетные распределения масс фрагментов. Видно, что количество фрагментов быстро уменьшается с увеличением их массы, модель хорошо воспроизводит экспериментальные данные. При разрушении приблизительно $72 \%$ от первоначальной массы цели составляют отдельные шарики. Большие фрагменты, состоящие из 25 или более стеклянных шариков, составляют всего около $2 \%$ массы.

Измеренные в лабораторных экспериментах двумерные поступательные скорости крупных фрагментов были в пределах от 1 до $13 \mathrm{~m} / \mathrm{s}$. Как и следовало ожидать, большие фрагменты двигались медленнее мелких. Для наблюдаемых фрагментов с массами порядка $3.5 \mathrm{~g}$ рассчитанная кинетическая энергия $0.3 \mathrm{~J}$ оказалась характерной для мелких фрагментов. Теоретическая модель также хорошо согласуется с наблюдаемой картиной, указывая на преобладание мелких фрагментов с кинетическими энергиями порядка $0.5 \mathrm{~J}$.

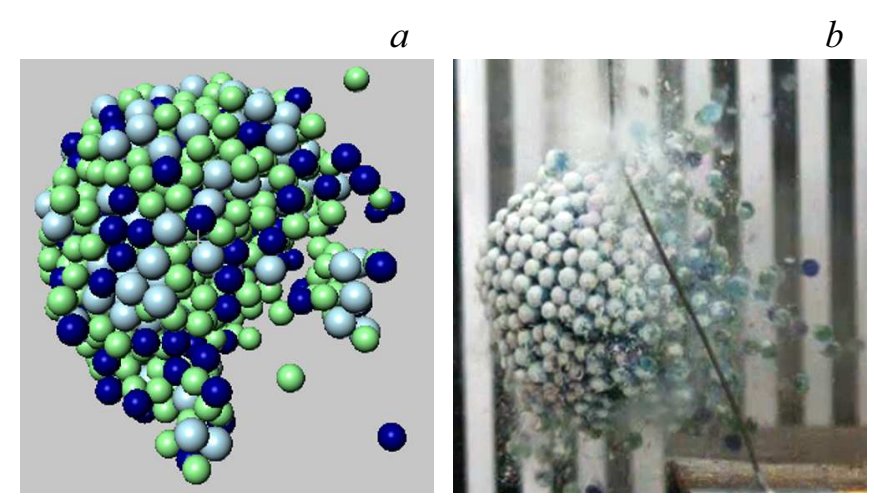

Рис. 6. Сравнение результата молекулярно-динамического расчета $(a)$ с экспериментом $(b)$. 


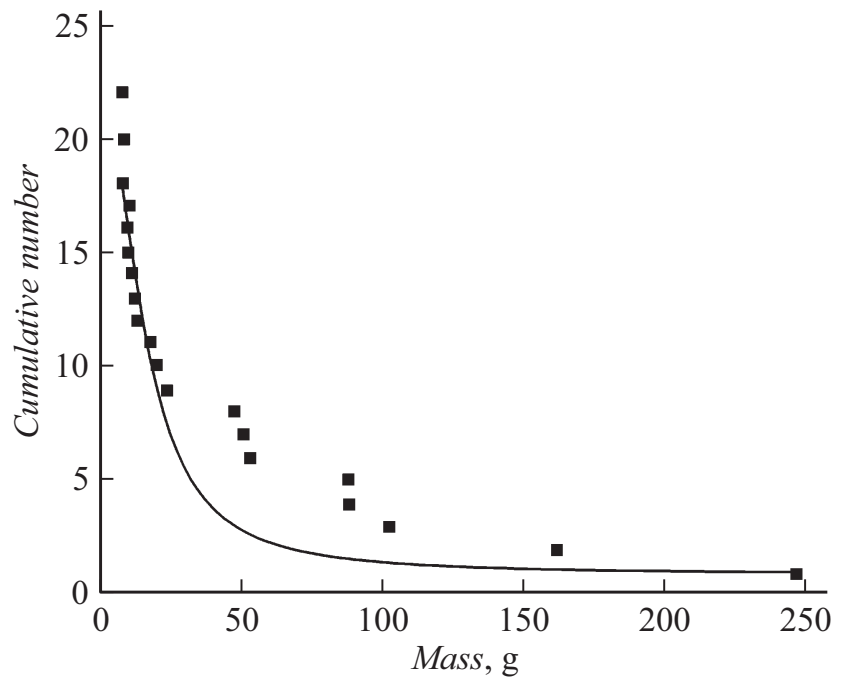

Рис. 7. Распределение масс фрагментов из эксперимента № 2 (точки) и из численной модели (кривая).

Важно отметить, что в модельных расчетах встречаются медленные фрагменты с массами, почти равными начальной массе мишени $(3.5 \mathrm{~kg})$. Мы можем провести оценку вероятности этих событий с учетом объема выборки МД-расчетов, как составляющую несколько десятых процента. Мы полагаем, что, выбирая начальные условия эксперимента и модельного расчета, можно управлять процессами образования фрагментов. В нашем эксперименте не было обнаружено тяжелых и медленных фрагментов мишени, что, естественно, объясняется небольшим количеством испытаний.

В заключении отметим, что описанные выше результаты относятся к ударам импакторов с высокой кинетической энергией, например, в эксперименте № 2 она составила $361 \mathrm{~J}$. Снижение уровня воздействия сохраняет мишень в исходном состоянии, что и было получено в результате наших экспериментов и расчетов, которые оказались в полном согласии.

Следующий эксперимент носил качественный характер. Моделировалось воздействие импульса излучения на поверхность тестового объекта, рассмотренного ранее. Импульс излучения заменялся воздействием на поверхность мелкой свинцовой дроби, движущейся со скоростью около $200 \mathrm{~m} / \mathrm{s}$. Условия выстрела подбирались таким образом, что пыж из патрона заведомо не попадал в объект, он тормозился в воздухе. Всего в наш объект попадало в среднем 100 дробинок, которые вносили энергию в поверхностный слой, и происходил вылет (испарение) фрагментов объекта в направлении обратном направлению движения дроби. В результате объект получал импульс, связанный с кинетической энергией дроби, а также импульс, определяемый вылетом из поверхности фрагментов мишени. В целом объект не разрушался, что важно для разработки методов его отклонения. Если из полного импульса, получаемого мишенью в результате выстрела, который измеряется в эксперименте, вычесть часть, связанную с кинетической энергией дроби (эта часть относительно просто вычисляется), то остается часть импульса, определяющая вылет фрагментов из объекта, которая, по мнению авторов, и моделирует действие импульсного излучения на объект.

Здесь надо отметить, что фрагменты содержали только целые стеклянные шарики, и значит энергия не расходовалась на их раскалывание. Целью этого эксперимента, проведенного в 2017 г., было показать, что мишень в целом сохраняет свою форму, не раскалываясь на несколько крупных фрагментов. Такой подход значительно более дешев, чем использованный в работе [5], и связан с более реалистичной моделью астероида.

Выбор оптимальных условий ударного воздействия на мишени требует дополнительных исследований, важная роль отводится модельным расчетам.

\section{Выводы}

Таким образом, для описания слабосвязанных сильно неоднородных систем представлен новый метод расчета, основанный на молекулярной динамике с потенциалом Л-Д. Для тестирования предложенной модели было проведено несколько экспериментов по лабораторному воздействию с использованием мишеней, состоящих из слабосвязанных стеклянных шариков в ледяной матрице. Для стрельбы использовались импакторы со скоростями в несколько сотен $\mathrm{m} / \mathrm{s}$, разрушение мишеней происходило по ледяным связям, практически без фрагментации стеклянных шариков. Достигнуто хорошее соответствие экспериментальных и численных результатов, что способствует дальнейшему развитию рассматриваемой модели. Одними из отличительных преимуществ подхода, рассматриваемого в работе, являются минимальные вычислительные затраты для проведения модельных расчетов. В отдельном эксперименте с рассредоточенным воздействием на мишень было показано, что не происходит хрупкого разрыва на большие фрагменты.

Настоящая работа является важным шагом в определении стратегий предотвращения потенциально опасных астероидов, особенно для разработки методов их отклонения без полного разрушения. В следующей работе будут реализованы несколько усовершенствований экспериментальной установки и модельных расчетов с целью лучшего воспроизведения реальных условий удара. Также будет изучаться использование потенциалов Л-Д при моделировании геологических материалов, аналогичных астероидам или кометам, которые не являются слабосвязанными.

\section{Конфрликт интересов}

Авторы заявляют, что у них нет конфликтов интересов. 


\section{Список литературы}

[1] Александров П.А., Горев В.В. // Природа. 2014. Вып. 5. C. 7-15.

[2] Ksanfomality L.V. // Phys. Uspekhi. 2012. Vol. 55. N 2. P. $137-146$.

[3] Wang G., Ostoja-Starzewski M. // Computational Mater. Sci. 2005. Vol. 33. P. 429-442.

[4] Krivtsov A.M., Pavlovskaia E.E., Wiercigroch M. „Impact Fracture of Rock Materials Due to Percussive Drilling Action", Proceedings of the XXI International Congress of Theoretical and Applied Mechanics (ICTAM 2004).

[5] Machii N., Nakamura A.M. // Icarus. 2011. Vol. 211. N 1. P. 885-893.

[6] Berezhnoi A. Glass-Ceramics and Photo-Sitalls. NY:: Plenum Press, 1970. $356 \mathrm{p}$.

[7] Fujiwara A., Cerroni P., Davis D., Ryan E., Di Martino M., Holsapple K., Housen K. Experiments and Scaling Laws for Catastrophic Collisions. In Asteroids II / Ed. R. Binzel M. Matthews T. Gehrels. Univ. of Arizona Press, 1989. P. 240-265.

[8] Schwatz S.R., Michel P., Richardson D.C. // Icarus. 2013. Vol. 226. N 1. P. 67-76.

[9] Luding S. // ESECE. 2008. N 12. P. 785-826.

[10] Tancredi G., Maciel A., Heredia L., Richeri P., Nesmachnow $S$. // Monthly Notices of the Royal Astronomical Society. 2012. Vol. 420. P. 3368-3380.

[11] Аристова Е. Ю., Аушев А.А., Баранов В.К. и др. // ЖЭТФ. 2018. Т. 153. Вып. 1. С. 157-172. 